\title{
Effects of pelvic stability on instep shooting speed and accuracy in junior soccer players
}

\author{
Ha-Rim Sung ${ }^{a}$, Won-Seob Shin ${ }^{a, b}$ \\ ${ }^{a}$ Department of Physical Therapy, Graduate School of Health and Medical, Daejeon University, Daejeon, Republic of Korea \\ ${ }^{b}$ Department of Physical Therapy, College of Health and Medical Science, Daejeon University, Daejeon, Republic of Korea
}

\begin{abstract}
Objective: The purpose of this study was to determine the effect of wearing a pelvic compression belt on ball speed and accuracy in instep shoots of youth soccer players.

Design: Randomized cross-over design.

Methods: We included 20 male junior soccer players with experience of more than 5 years. Participants were randomly assigned to two conditions: application of a pelvic compression belt and instep shooting or no application. Instep shooting was performed three times at a distance of 20 meters from the position of the goal post, and the ball speed was measured using a speed gun at a position 5 meters behind the goal post. The shooting accuracy was measured based on a 5-point scale. The shooting accuracy was measured by scoring 5 points at 2.44 meters in the middle of the goal area of area A, 3 points at 2.44 meters in the goal area of area $\mathrm{B}$, and 0 in the case of shooting outside the goal area C.

Results: After applying a pelvic compression belt, the mean speed of the ball was significantly increased $(p<0.05)$. The maximum speed of the ball was significantly increased $(p<0.05)$. The accuracy of the ball was significantly increased $(p<0.05)$.

Conclusions: Through this study, we expect that the use of the pelvic compression belt can be applied as a training method to improve the shooting ability of soccer players. Clinically, pelvic compression belts are expected to help rehabilitation soccer players to improve their shooting accuracy.
\end{abstract}

Key Words: Pelvis, Pressure, Puberty, Soccer

\section{Introduction}

Soccer is a game in which the ball is placed into the opponent's goal, and the head, the foot, and the body is used as a means of making a goal; however, a shoot made by the foot constitutes most of the goal. That is, foot shoots are the most frequently performed technique and is considered to be the core of soccer games [1]. Shooting is an important part of the soccer game, and therefore, its operation must be strong and accurate.

Among these, instep shooting is a shooting action that uses the wide area of the foot, and most kinematics researchers report that the kick in a soccer kick is a better kick than other types of kicks for maximum ball speed and accuracy [2]. Therefore, the improvement of instep kick performance is recognized as one of the most important goals in the training process of young soccer players, and practicing shooting techniques in soccer is the first step in improving soccer performance [3]. Previous studies have compared and analyzed the kinematics of the instep shooting motion. This study focuses on the correlation between instep shooting, lower limb motion, and the lower extremity muscles, and discusses the important role of the lower extremity muscles in the shooting motion [4]. Among them, some studies have analyzed the limb segments of male and female soccer players in terms of kinematics from the kick instinct kicking

Received: 21 May, 2018 Revised: 4 June, 2018 Accepted: 10 June, 2018

Corresponding author: Won-Seob Shin (ORCID http://orcid.org/0000-0002-6515-7020)

Department of Physical Therapy, Graduate School of Health and Medical, Daejeon University, 62 Daehak-ro, Dong-gu, Daejeon 34520, Republic of Korea Tel: 82-42-280-2290 Fax: 82-42-280-2295 E-mail: shinws@dju.kr

(c) This is an Open-Access article distributed under the terms of the Creative Commons Attribution Non-Commercial License (http://creativecommons.org/licenses/ by-nc/4.0) which permits unrestricted non-commercial use, distribution, and reproduction in any medium, provided the original work is properly cited.

Copyright $\odot 2018$ Korean Academy of Physical Therapy Rehabilitation Science 
operation and presented the kinematic data of the supporting foot during the kicking operation. According to the results, in order to increase the ball's speed, it is important to position the supporting foot side-by-side with the ball and increase the foot speed immediately before the impact. In the downswing phase, the overall swing pattern contributes significantly to the hip. It is emphasized that the timing of the knee joint leading to the other knee joint is the time immediately before the coinciding of both knees, which is the latter half of the swing, to the time immediately before the impact.

In addition, studies on fitness and shooting performance and studies on leg strength have been focused. The results of the previous studies show that rather than only the leg strength, both leg and core strength of the human body interact with each other and have an important influence on the fitness and shooting performance. Among the preceding articles related to the core, the abdomen and the waist region in the human body is called the power zone, thereby allowing the limb to exert its strength and make it stronger, and the core muscle in the center of the body is stimulated by the movements of the limb. In order for one part of the body to function effectively, the other part must function as a fixed point so that it can perform effectively in sports activities. In order to maintain the functional stability of the body, it is said that the control of the muscles around the lumbar region plays an important role [5]. If the state of the core muscle is good, the energy of the trunk can be transmitted to the limbs. As optimal state of the core muscle promotes trunk stabilization and enhanced movement of the limbs [6]. Core programs affect the isokinetic strength, flexibility, and balance ability of youth soccer players with chronic back pain. Core exercises are very beneficial exercises for athletes who maintain balance and agility through balanced stability of the pelvis [7].

Recently, pelvic compression belts (PCB) have been used in clinical practice to provide external stabilization of the pelvis by providing compression on the sacroiliac (SI) joints and as a tool for restoring stability of the stabilizing muscles of the trunk and the SI joint [8]. In fact, the application of an external pelvic compression belt to persons with low back pain have been reported to be effective in reducing symptoms by reducing the stress placed onto the pain-sensitive structures through increasing the compressive force in the SI joint and promoting the stability of the pelvis [9]. The sufficient activity of the muscles around the SI joint plays an important role in the regulation of joint stability and movement [10]. In order to maintain the stability of the SI joints, coordi- nation between the muscles by the control of the appropriate nervous system is required [11]. The pelvic compression belt affects pelvic motility [12] and applies pressure onto the pelvis, which increases the abdominal pressure, and the muscular activity of the pelvis during movement may provide additional proprioceptive stimulation to the gluteal muscle through compression [13].

Therefore, the purpose of this study was to determine the effect of the use of a pelvic compression belt on ball speed and accuracy in instep shoots of youth soccer players.

\section{Methods}

\section{Participants}

This study was a part of the soccer federation of the Korea Football Association conducted with 23 male junior soccer players with an experience of 5 or more years. Participants were selected from the preliminary questionnaires who have not been diagnosed with injuries to the back, knee, or ankle within the past 6 months, and who did not have any pain with the shooting motion. Three out of 23 subjects felt pain during shooting due to back and ankle pain and were excluded from the study. A total of 20 subjects participated in the experiment. All subjects were fully informed about the methodology of the instep shooting and the purpose of the study, and those who voluntarily agreed to participate in the experiment were included. This study was conducted with the approval of the Institutional Review Board of the Daejeon University (IRB No. 1040647-201805-HR-002-03).

\section{Procedure}

This study is a randomized cross-over design study. Subjects were given two colored cards in which they randomly drew in order to determine their order. The red color indicated no application of a pelvic compression belt and the blue color indicated application of a pelvic compression belt and instep shooting. The pelvic compression belt (The Com-Pressor Belt; OPTP, Minneapolis, MN, USA) was used to provide a compression force to the left and right SI joint (Figure 1). The belt consisted of a body belt and a compression band, which was applied directly under the anterior superior iliac spine to compress the entire pelvis.

Instep shooting was performed three times at a distance of 20 meters from the goal post (Figure 2), and the ball speed was measured by using a speed gun at a position 5 meters behind the goal post. The average value of the maximum speed record and the three full speed recordings were used as the 


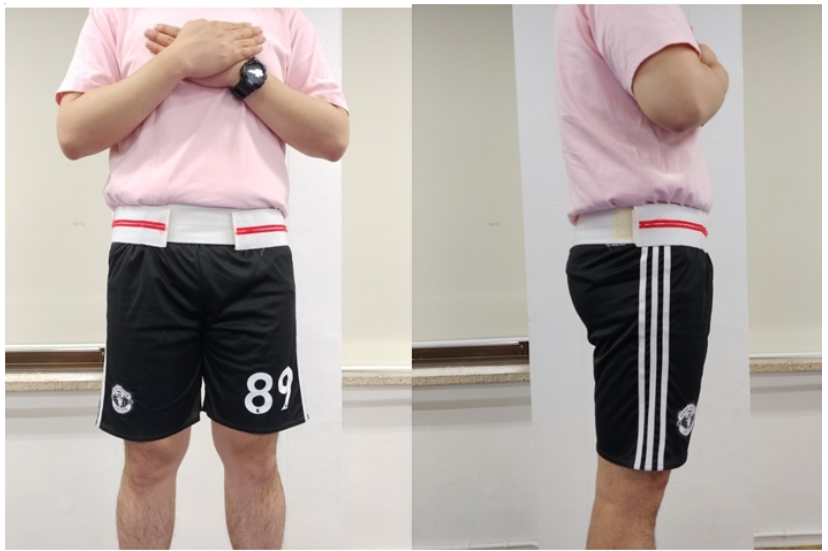

Figure 1. Pelvic compression belt.

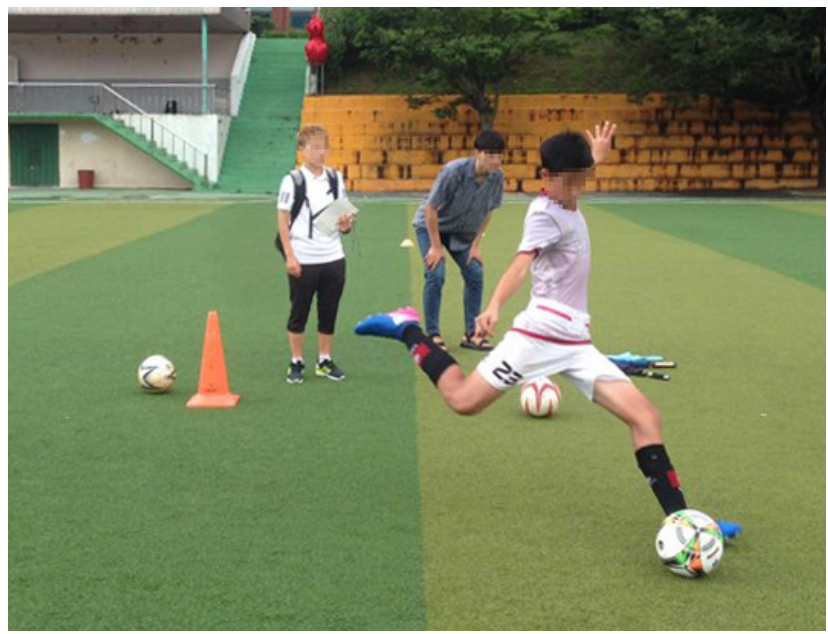

Figure 2. Instep shooting wearing a compression belt.

measurement values of the present study [14].

\section{Outcome measures}

\section{Shooting speed}

The speed gun (Velocity Speed Gun; Bushnell, Overland Park, KS, USA) has a minimum measuring error of \pm 1 to 2 $\mathrm{km} / \mathrm{h}$. Since changing the position of the speed gun at every measurement period could affect the results, measurements were performed in the same position behind the goal post. These distances ensured that the direction of motion of the ball was less than 5 degrees from the straight line when the gun was oriented horizontally towards the server, reducing "cosine" errors to less than $0.5 \%$.

\section{Shooting accuracy}

The shooting accuracy was measured by scoring 5 points at 2.44 meters in the middle of the goal area of area A, 3
Table 1. General characteristics of subjects

\begin{tabular}{lr}
\hline \multicolumn{1}{c}{ Variable } & \multicolumn{1}{c}{ Value } \\
\hline Age $(\mathrm{y})$ & $15.65(0.59)$ \\
Height $(\mathrm{cm})$ & $161.75(6.05)$ \\
Weight $(\mathrm{kg})$ & $50.35(7.25)$ \\
Career $(\mathrm{y})$ & $5.75(0.44)$ \\
Dominant foot & \\
Left & 2 \\
Right & 18 \\
Position & 13 \\
Strikers & 6 \\
Defenders & 1 \\
Goal keeper & \\
\hline
\end{tabular}

Values are presented as mean (SD) or number only.

points in the goal area 2.44 meters of area $\mathrm{B}$, and 0 points in the case of shooting outside the goal area C [15].

\section{Data and statistical analysis}

The information obtained from the measurements was analyzed using IBM SPSS Statistics ver. 22.0 (IBM Co., Armonk, NY, USA). The general characteristics of the subjects were presented using the mean and standard deviation using descriptive statistics and the Kolmogorov-Smirnov normality test was conducted. A paired-t test was used to compare the speed and accuracy of shoots with and without pelvic compression belt. To investigate the effect of use of a compression belt in each condition, the paired t-test was conducted. Statistical significant was set at 0.05 .

\section{Results}

\section{General characteristics of subjects}

The subjects of this study were Eunhye Middle School male soccer players, with 18 who were right-foot-dominant, and 2 who were left-foot-dominant. Their positions were 13 strikers, 6 defenders and 1 goalkeeper.

The mean age of the subjects was 15.65 years, mean height was $161.75 \mathrm{~cm}$, mean weight was $50.35 \mathrm{~kg}$, and average career was 5.75 years. The characteristics of the subjects of this study are shown in Table 1.

\section{Comparison of shooting speeds}

The mean speed was $75.42 \pm 8.19 \mathrm{~km} / \mathrm{h}$ when the pelvic compression belt was not worn and was $80.22 \pm 7.52 \mathrm{~km} / \mathrm{h}$ when wearing the pelvic compression belt, which was significantly increased by $4.80 \pm 1.98 \mathrm{~km} / \mathrm{h}(p<0.05)$. 
Table 2. Comparison of shooting speed and accuracy with compression belt application

$(\mathrm{N}=20)$

\begin{tabular}{lcccc}
\hline \multicolumn{1}{c}{ Variable } & Wearing a belt & Not wearing a belt & $\mathrm{t}$ & $p$-value \\
\hline Mean speed $(\mathrm{km} / \mathrm{h})$ & $80.22(7.52)$ & $75.42(8.19)$ & 10.841 & $<0.001$ \\
Maximum speed $(\mathrm{km} / \mathrm{h})$ & $82.40(8.42)$ & $77.80(8.48)$ & 8.519 & $<0.001$ \\
Accuracy (score) & $4.07(0.61)$ & $2.95(1.02)$ & 4.721 & $<0.001$ \\
\hline
\end{tabular}

Values are presented as mean (SD).

The maximum speed was $77.80 \pm 8.48 \mathrm{~km} / \mathrm{h}$ when the pelvic compression belt was not worn, and $82.40 \pm 8.42 \mathrm{~km} / \mathrm{h}$ when wearing the pelvic compression belt, which was significantly increased by $4.60 \pm 2.41 \mathrm{~km} / \mathrm{h}(p<0.05$; Table 2$)$.

\section{Comparison of shooting accuracy}

The accuracy score was $2.95 \pm 1.02$ points when the pelvic press belt was not worn and was $4.07 \pm 0.61$ points when wearing the pelvic compression belt, which was significantly increased by $1.13 \pm 1.07$ points $(p<0.05$; Table 2$)$.

\section{Discussion}

This study was conducted to investigate the influence of the application of a pelvic compression belt on shooting performance (accuracy, speed) of middle school soccer players.

In the results of this study, the accuracy, average speed, and maximum speed of instep shooting were improved significantly according to whether the pelvic compression belt was worn or not. The average and maximum speed was significantly improved. According to the study of Jovanovic et al. [16], strength, power, agility, and flexibility are required to be influential factors and physical coordination ability is required. In addition, Gonçalves and Pereira [17] reported that pelvic fixation during dynamic trunk extension exercise induces higher muscle activity in the trunk extensors, suggesting that highly efficient training is possible in other areas. It is believed that application of the pelvic compression belt enhances the movement of the torso or lower extremity by providing stabilization. Therefore, during the shooting motion, pelvic compression will stabilize the pelvis and enable the muscles involved in shooting to become more active. In addition, according to a study by Lee [18], dynamic balance is increased when the pelvic compression belt is worn, has a positive effect on the muscle activity of the trunk related to the pelvic motility and stability, and contributes to the external stabilization of the pelvis. There was no statistically significant difference in leg strength between the two groups, but there was an increase in the balance of individuals with stroke and the strength of the lower legs [19].

In conclusion, when the pelvic compression belt was worn, it stabilized the trunk and increased the muscle activity of the lower limbs, and therefore, the average and maximum speeds were significantly increased. The shooting accuracy was also improved. At the time of the experiment, the players had muscle fatigue after training for more than 8 hours. At the time of the experiment, the players had muscle fatigue after training for more than 8 hours. Since this is similar to the real case of where the exhausted soccor player performs a penalty shoot-out after playing an actual soccor game, the shooting measurement results of this study are considered to be of value. Long-term exercise and training results in a decrease in the ability to produce maximum muscle strength and thus, muscle fatigue changes the muscle contraction efficiency and proprioception. It is considered that training or competing for long periods of time may affect motor coordination and postural control and subsequently lead to joint or muscle deterioration [20].

Therefore, it plays an important role in distributing and transferring the weight load to the place where the center of gravity of the body is located in the SI joint and the pelvis [8]. Stability should be provided to the SI joints and pelvis to maintain alignment and dynamic balance of postures while performing functional activities, such as beginning of movement or walking and running. Previous studies have shown that the proximal movement of the trunk are slow when limb movements are slow [21] due to low multifidus muscle activity, but when the limb movements are accelerated, the activity of the multifidus muscles increases.

The pelvic compression belt is a tool that provides a certain amount of pressure [22], and relieves pain related to pelvic instability by increasing the passive stability of the pelvis and promotes pain reduction in patients with unstable lower backs and SI joints [23]. The study showed that the stability of the SI joint and the pelvic girdle was improved with application of a pelvic compression belt, which improved the performance of functional activities such as shooting, and 
shooting accuracy was also improved.

As a limitation of this study, subjects were middle school soccer players and consisted only male gender players and therefore, it may be difficult to generalize the results to various age groups and female soccer players. Therefore, future studies should include soccer players with back pain, subjects of various ages, and female soccer players as subjects in order to further investigate the effects of applying a pelvic compression belt.

In conclusion, the application of a pelvic compression belt is considered to be an effective way to improve the performance of shoots in instep shoots. Clinically, PCB are expected to help soccer players improve their shooting accuracy during rehabilitation.

\section{Conflict of Interest}

The authors declared no potential conflicts of interest with respect to the authorship and/or publication of this article.

\section{References}

1. Song SG. Analysis of correlation between shooting speed and isokinetic strength in middle school soccer players [Master thesis]. Seoul: Sejong University; 2003.

2. Beckenbauer F. Franz Beckenbauer's soccer power techniques, tactics, training. New York: Simon and Schuster; 1987. p. 117-8.

3. Weineck J. Coaching soccer-conditioning. Thessaloniki: Edition Salto; 1997.

4. Lees A, Asai T, Andersen TB, Nunome H, Sterzing T. The biomechanics of kicking in soccer: a review. J Sports Sci 201028:805-17.

5. Kim KJ. Effects of core muscle strengthening training on flexibility, muscular strength and driver shot performance in female professional golfers. Int J Appl Sport Sci 2010;22:111-27.

6. Akuthota V, Nadler S F. Core strengthening. Arch Phys Med Rehabil 2004;85(3 Suppl 1):S86-92.

7. Jo YH. Effect of isotonic exercise program and core exercise program on isokinetic muscular strength, flexibility, balance, and visual analog scale of teenage soccer player [Master thesis]. Seoul: Kyung Hee University; 2010.

8. Pel JJ, Spoor CW, Goossens RH, Pool-Goudzwaard AL. Biomechanical model study of pelvic belt influence on muscle and ligament forces. J Biomech 2008;41:1878-84.

9. Beales DJ, O'Sullivan PB, Briffa NK. The effects of manual pelvic compression on trunk motor control during an active straight leg raise in chronic pelvic girdle pain subjects. Man Ther 2010; 15:190-9.

10. Hungerford B, Gilleard W, Hodges P. Evidence of altered lumbopelvic muscle recruitment in the presence of sacroiliac joint pain. Spine (Phila Pa 1976) 2003;28:1593-600.

11. Sjödahl J, Kvist J, Gutke A, Öberg B. The postural response of the pelvic floor muscles during limb movements: a methodological electromyography study in parous women without lumbopelvic pain. Clin Biomech (Bristol, Avon) 2009;24:183-9.

12. Mens JM, Damen L, Snijders CJ, Stam HJ. The mechanical effect of a pelvic belt in patients with pregnancy-related pelvic pain. Clin Biomech (Bristol, Avon) 2006;21:122-7.

13. de Groot M, Pool-Goudzwaard AL, Spoor CW, Snijders CJ. The active straight leg raising test (ASLR) in pregnant women: differences in muscle activity and force between patients and healthy subjects. Man Ther 2008;13:68-74.

14. Andersen TB, Dörge HC. The influence of speed of approach and accuracy constraint on the maximal speed of the ball in soccer kicking. Scand J Med Sci Sports 2011;21:79-84.

15. Choi JH, Park YH. Effects of core training on physical fitness and shooting performance ability in the middle school soccer player. J Phys Educ Lifetime Sport Sci 2014;26:59-76.

16. Jovanovic M, Sporis G, Omrcen D, Fiorentini F. Effects of speed, agility, quickness training method on power performance in elite soccer players. J Strength Cond Res 2011;25:1285-92.

17. Gonçalves M, Pereira MP. Effect of a pelvic belt on EMG activity during manual load lifting. BrazJ Kinanthropometry Hum Perform 2009;11:151-9.

18. Lee JE, Lee CH, Kwon OY, Park SY. Dynamic balance and muscle activity of the trunk and hip extensor following the wearing of pelvic compression belt. Phys Ther Korea 2015;22:49-57.

19. Kim YH. The effects of trunk stability exercise on the balance and gait in the patients with stroke [Master thesis]. Daegu: Daegu University; 2010.

20. Allen DG, Westerblad H. Role of phosphate and calcium stores in muscle fatigue. J Physiol 2001;536:657-65.

21. Palluel E, Ceyte H, Olivier I, Nougier V. Anticipatory postural adjustments associated with a forward leg raising in children: effects of age, segmental acceleration and sensory context. Clin Neurophysiol 2008;119:2546-54.

22. Haugland KS, Rasmussen S, Daltveit AK. Group intervention for women with pelvic girdle pain in pregnancy. A randomized controlled trial. Acta Obstet Gynecol Scand 2006;85:1320-6.

23. Liebenson $\mathrm{C}$. The relationship of the sacroiliac joint, stabilization musculature, and lumbo-pelvic instability. J Bodyw Mov Ther 2004;8:43-5. 